\title{
artigo
}

Oliveira, C.R.; Ribeiro, T.S.; Dellanhese, A.P.F.

Medidas de prevenção e controle da tuberculose às pessoas privadas de liberdade em presídios brasileiros

\section{Medidas de prevenção e controle da tuberculose às pessoas privadas de liberdade em presídios brasileiros}

\author{
Measures for the prevention and control of tuberculosis to persons deprived of freedom in brazilian presidents
} Medidas para la prevención y control de la tuberculosis a personas privadas de libertad en presidentes brasileños

\section{RESUMO}

OBJETIVO: Identificar medidas de prevenção e controle da tuberculose (TB) a Pessoas Privadas de Liberdade (PPL) e que vivem com TB nos presídios brasileiros. MÉTODO: Trata-se de uma revisão integrativa, março a dezembro de 2018. As bases de dados utilizadas foram: SciELO, LILACS e MEDLINE, a partir dos descritores Atenção à saúde; Tuberculose; Prisão. Foram incluídos estudos, publicados entre 2012 e 2018, em português, inglês e espanhol com o Brasil como país de origem e artigos originais completos. RESULTADOS: Foram encontrados 181 estudos, após exclusões 2 estudos foram analisados. Os principais fatores abordados pelos artigos selecionados trazem as políticas públicas voltadas às PPL quanto às formas de prevenção e controle da TB nos presídios brasileiros. CONCLUSÃO: Detectou-se que a implementação das políticas públicas voltadas ao controle da TB no sistema prisional é realizada de forma parcial. Foi identificado a carência de estudos relativos às medidas de prevenção e controle da TB nas prisões brasileiras.

DESCRITORES: Atenção à saúde; Tuberculose; Prisão;

\section{ABSTRACT}

OBJECTIVE: To identify tuberculosis (TB) prevention and control measures for people deprived of liberty (PPL) and living with TB in Brazilian prisons. METHOD: This is an integrative review, from March to December 2018. The databases used were: SciELO, LILACS and MEDLINE, based on the keywords Health care; Tuberculosis; Prison. Studies, published between 2012 and 2018 , in Portuguese, English and Spanish with Brazil as the country of origin and complete original articles were included. RESULTS: 181 studies were found, after exclusions 2 studies were analyzed. The main factors addressed by the selected articles bring public policies aimed at the PLP regarding the forms of TB prevention and control in Brazilian prisons. CONCLUSION: It was found that the implementation of public policies aimed at TB control in the prison system is partially carried out. The lack of studies related to TB prevention and control measures in Brazilian prisons was identified.

DESCRIPTORS: Health Care; Tuberculosis; Prisons;

\section{RESUMEN}

OBJETIVO: Identificar medidas de prevención y control de la tuberculosis (TB) para las personas privadas de libertad (PPL) y que viven con TB en las cárceles brasileñas. MÉTODO: Se trata de una revisión integradora, de marzo a diciembre de 2018. Las bases de datos utilizadas fueron: SciELO, LILACS y MEDLINE, con base en las palabras clave Health care; Tuberculosis; Prisión. Se incluyeron estudios, publicados entre 2012 y 2018, en portugués, inglés y español con Brasil como país de origen y artículos originales completos. RESULTADOS: Se encontraron 181 estudios, luego de las exclusiones se analizaron 2 estudios. Los principales factores abordados por los artículos seleccionados traen las políticas públicas dirigidas al PLP sobre las formas de prevención y control de la TB en las cárceles brasileñas. CONCLUSIÓN: Se encontró que la implementación de políticas públicas orientadas al control de la TB en el sistema penitenciario se realiza parcialmente. Se identificó la falta de estudios relacionados con las medidas de prevención y control de la tuberculosis en las cárceles brasileñas.

DESCRIPTORES: Atención a la Salud; Tuberculosis; e Prisiones.

RECEBIDO EM: 08/09/2020 APROVADO EM: 17/09/2020

\section{Cláudia Rodrigues de Oliveira}

Bacharel em Enfermagem pelo Centro Universitário Ritter dos Reis (UniRitter - Laureate International Universities). Pós-Graduanda MBA em Auditoria em Saúde pela Faculdade de Tecnologias em Saúde (Fasaude). Graduanda em Saúde Coletiva pela Universidade Federal do Rio Grande do Sul (UFRGS), Enfermeira Pesquisadora Voluntária de Iniciação Científica pela Universidade Federal do Rio Grande do Sul. Enfermeira da Unidade de Saúde Vila Jardim pelo Instituto de Cardiologia, Rio Grande do Sul (IC-FUC/RS), Porto Alegre-RS.

ORCID: 0000-0003-0074-9495 


\section{Tayná da Silva Ribeiro}

Bacharel em Enfermagem pelo Centro Universitário Ritter dos Reis (UniRitter - Laureate International Universities). Pós-graduanda em Saúde Pública pela Universidade Federal do Rio Grande do Sul. Enfermeira Pesquisadora voluntária pela Universidade Federal do Rio Grande do Sul. Enfermeira da Unidade de Saúde Campo Novo da APS Vila Nova, Porto Alegre-RS. ORCID: 0000-0002-5418-631X

\section{Amanda Pereira Ferreira Dellanhese}

Enfermeira da Unidade de Saúde Mapa da Gerência Distrital Partenon, Lomba do Pinheiro da APS Hospital Divina Providência, Porto Alegre-RS. Doutora em Saúde da Criança e do Adolescente pela Universidade Federal do Rio Grande do Sul. Docente Colaboradora da Residência Integrada Multiprofissional em Saúde Coletiva da Universidade Federal do Rio Grande do Sul. ORCID: 0000-0002-1515-9693

\section{INTRODUÇÃO}

A s Pessoas Privadas de Liberdade (PPL) representam um sério problema de saúde pública, no que diz respeito ao controle de doenças infectocontagiosas como a tuberculose $(\mathrm{TB})^{1}$. O perfil das PPL que vivem com TB no Brasil, trata-se de uma população jovem, predominantemente masculina, negra, com baixa escolaridade, baixo poder aquisitivo, co-infectada por HIV/ AIDS ${ }^{2}$. Santos (2014) relata as condições de saúde em que as PPL são submetidas, expostas a ambientes superlotados e mal ventilados, com má nutrição, fazendo consumo de drogas, convivendo com serviços de saúde precários ou inexistentes, fatores determinantes para o alto índice de TB nos presídios brasileiros $^{3}$. O crescimento populacional no cárcere e as questões ligadas à desigualdade social vêm tencionando as instituições responsáveis para a elaboração de políticas públicas que visem melhorar as condições de vida no sistema prisional ${ }^{4}$.

O Ministério da Saúde (MS) recomenda estratégias de prevenção e controle da TB que contemplam as PPL. Há obstáculos que dificultam a implementação de estratégias de prevenção e controle da TB nos presídios brasileiros, como a estigmatização e segregação, falta de recursos humanos e financeiros, inadequação dos serviços de saúde, restrição da autonomia das PPL com baixa participação no tratamento e nas ações de prevenção e a falta de informação sobre TB 5 . O Programa Nacional de Controle da Tuberculose (PNCT), criado em 2006, tem como objetivo desenvolver estratégias relacionadas à descentralização e horizontalização ${ }^{6}$. O programa reconhece a importância dessas estratégias para o combate à $\mathrm{TB}$, estendendo-as para todos os serviços de saúde do Sistema Único de Saúde - SUS 5 .

"O PNCT está integrado na rede de Serviços de Saúde. É desenvolvido por intermédio de um programa unificado, executado em conjunto pelas esferas federal, estadual e municipal. Está subordinado a uma política de programação das suas ações com padrões técnicos e assistenciais bem definidos, garantindo desde a distribuição gratuita de medicamentos e outros insumos necessários até ações preventivas e de controle do agravo. Isto permite o acesso universal da população às suas ações." ${ }^{6}$

Nas últimas três décadas, o Estado desenvolveu políticas específicas para as PPL, Lermen et al (2015) traz em seus estudos as políticas voltadas à população carcerária, sendo elas: a Lei de Execução Penal (LEP) ${ }^{7}$, que garante os direitos de saúde nos estabelecimentos prisionais. A LEP prevê atendimento em saúde a toda a população prisional, seja ela provisória ou apenada, compreendendo atendimento médico, farmacêutico e odontológico; o Plano Nacional de Saúde no Sistema Penitenciário - PNSSP ${ }^{8}$, que legitima a garantia de acesso à saúde das PPL, sua proposta é garantir ações integrais de saúde, além da assistência à prevenção e à promoção da saúde da população carcerária; a Política Nacional de Atenção Integral à Saúde das Pessoas Privadas de Liberdade no Sistema Prisional - PNAISP ${ }^{9}$, que tem como objetivo contribuir para o controle e ou redução dos agravos de saúde mais frequentes da população carcerária brasileira, bem como trabalhar na lógica de prevenção de doenças e da promoção da saúde.

Diante do cenário brasileiro atual, relacionado ao crescimento populacional no cárcere e a situação endêmica da $\mathrm{TB}$ a nível nacional, torna-se importante compreender como que tem sido desenvolvida a assistência à saúde às pessoas privadas de liberdade e que vivem com TB. O presente estudo tem como objetivo de identificar medidas de prevenção e controle da tuberculose (TB) a Pessoas Privadas de Liberdade (PPL) e que vivem com $\mathrm{TB}$ nos presídios brasileiros.

\section{MÉTODO}

Trata-se de uma revisão da literatura, do tipo integrativa, visto que o estudo permite a análise crítica das pesquisas que foram selecionadas, possibilitando a interpretação e a síntese dos dados coletados, além de apontar lacunas do conhecimento que precisam ser preenchidas com a realização de novos estudos, baseando-se por seis etapas: 1) identificação do tema e seleção da hipótese ou questão de pesquisa para a elaboração da revisão integrativa; 2) estabelecimento de critérios para inclusão e exclusão de estudos/ amostragem ou busca na literatura; 3) definição das informações a serem extraídas dos estudos selecionados/ categorização dos estudos; 4) avaliação dos estudos incluídos na revisão integrativa; 5) interpretação dos resultados e 6) apresentação da revisão/síntese do conhecimento(10). Foi estabelecida a 
pergunta norteadora do estudo: "Quais medidas de prevenção e controle da tuberculose (TB) a Pessoas Privadas de Liberdade (PPL) e que vivem com TB nos presídios brasileiros?" que auxilia no processo de realização das atividades, incluindo também a definição dos participantes, das intervenções e dos resultados que almejam ser alcançados ${ }^{11}$.

A busca de artigos foi realizada no período de setembro de 2018 nas seguintes bases de dados eletrônicas Scientific Electronic Library Online (SciELO); Literatura Latino-Americana e do Caribe em Ciências da Saúde (Lilacs); e Medical Literature Analysis and Retrieval System Online (Medline). Os descritores foram definidos pela biblioteca virtual em saúde DeCS (Descritores em Ciência da Saúde): Atenção à saúde; Tuberculose; Prisão; Health Care; Tuberculosis; Prisons; Atención a la Salud; Tuberculosis; e Prisiones. Para a combinação desses DeCs, utilizou-se os opera-

Figure 1 - Flowchart of the stages of study and selection of articles in the SciELO, LILACS and MEDLINE databases

$1^{\circ}$ etapa: definição da pergunta problema

"Como funciona a atenção a saúde de pessoas privadas de liberdade que vivem com tuberculose nos presídios brasileiros?"

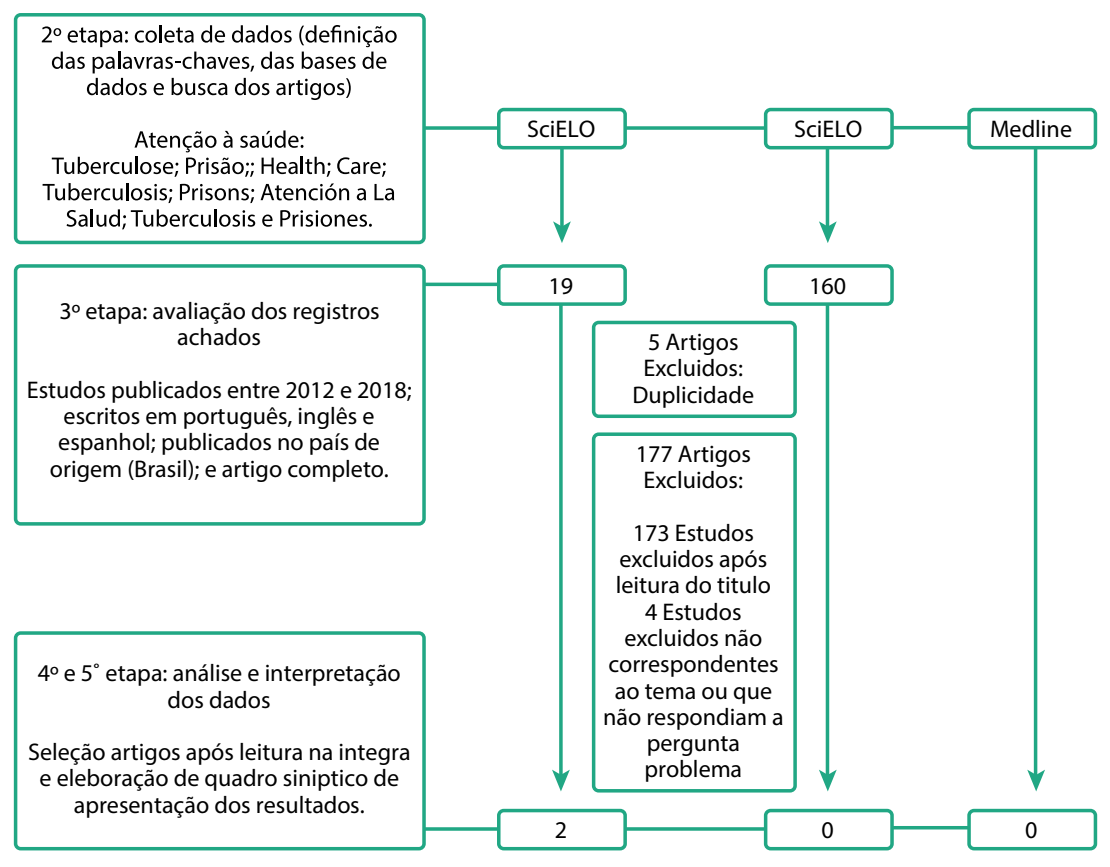

leitura na íntegra, sendo 2 artigos retirados por duplicidade. A amostra final desta revisão foi composta por 2 artigos. Os estudos foram sintetizados de forma a apresentar as medidas de prevenção e controle da TB e a implementação das políticas de saúde voltadas às PPL.

\section{RESULTADOS}

Os dados obtidos foram agrupados e apresentados em quadro, de modo a possibilitar uma melhor visualização dos estudos inseridos na revisão integrativa. Foram inseridas as informações: autor/ ano; tipo de estudo; e revista; Ações de prevenção e controle da Tuberculose nos presídios brasileiros (Quadro 1). Os estudos tratam-se de casos múltiplos com abordagem qualitativa e estudo de múltiplos discursos com fundamento referencial teórico-metodológico da análise de discurso de matriz francesa. Alguns fatores, no que diz respeito às medidas de prevenção e controle da TB, foram mais citados: o PNCT, PNSSP e PNAISP. Além disso, trazem instrumentos usados na prevenção e no controle da doença dentro do sistema prisional, a baciloscopia de escarro, radiografia, prova tuberculina, Teste de Sensibilidade ao Antimicrobiano (TSA), testagem anti-HIV, notificação e TDO.

\section{DISCUSSÃO}

As condições de saúde das PPL são relacionadas a questôes sociais como: população jovem, com baixa escolaridade, baixo poder aquisitivo e provenientes de regiões com alta incidência de TB. Além disso, é apresentada a questão da superlotação de celas e do confinamento em locais mal ventilados, com más condições sanitárias, fatores que levam à disseminação da doença. Valença, Possuelo, Cezar-Vaz e Silva (2016) relatam que a TB no âmbito prisional está associada a características individuais e a condição de vida do sujeito antes do encarceramento, bem como a fatores ambientais relacionados 
Chart 1 - Characterization of studies according to authorship, year of publication, type of study and Tuberculosis prevention and control actions in Brazilian prisons

\begin{tabular}{|c|c|c|c|}
\hline ANO & PERIÓDICO & TIPO DE ESTUDO & $\begin{array}{l}\text { A ATENÇÃO À SAÚDE DE PPL QUE VIVEM COM TB NOS PRESÍDIOS } \\
\text { BRASILEIROS }\end{array}$ \\
\hline $2015^{12}$ & $\begin{array}{l}\text { Cadernos de Saú- } \\
\text { de Pública. }\end{array}$ & $\begin{array}{l}\text { Estudo de casos múl- } \\
\text { tiplos com abordagem } \\
\text { qualitativa. }\end{array}$ & $\begin{array}{l}\text { as ações do PNCT são parcialmente implementadas nas prisões estudadas } \\
\text { nos dois dos estados com maior população prisional do Brasil e com altas ta- } \\
\text { xas de incidência de TB. No Caso 1, a Unidade de Análise } 1 \text { (UA1) e Unidade de } \\
\text { Análise } 2 \text { (UA2) dispõe de exame de ingressos, busca ativa dos sintomáticos } \\
\text { realizados de forma sistemática (campanhas) e o TDO, realizado por profis- } \\
\text { sionais de saúde. No Caso 2, os autores apresentam que o Hospital Penal } \\
\text { (UA3), unidade onde foram implementadas ações para prevenção e contro- } \\
\text { le da doença, dispunha de mais recursos humanos, exames laboratoriais e } \\
\text { radiológicos. Ainda no Caso 2, a Unidade de Análise } 4 \text { (UA4) e Unidade de } \\
\text { Análise } 5 \text { (UA5) utilizam o rastreamento radiológico, que apresentou melhor } \\
\text { desempenho, inclusive na deteç̧ão de suspeitos assintomáticos. No que diz } \\
\text { respeito à entrega dos medicamentos em ambos os casos, a função é reali- } \\
\text { zada por profissionais de saúde durante a semana e no final de semana pelo } \\
\text { agente de segurança, sendo o tratamento auto administrado. }\end{array}$ \\
\hline $2012^{13}$ & $\begin{array}{l}\text { Texto \& Contexto } \\
\text { Enfermagem. }\end{array}$ & $\begin{array}{l}\text { Estudo de múltiplos } \\
\text { discursos com } \\
\text { fundamento referencial } \\
\text { teórico-metodológico da } \\
\text { análise de discurso de } \\
\text { matriz francesa. }\end{array}$ & $\begin{array}{l}\text { O estudo traz que os direitos constitucionais à saúde das PPL, nas unidades } \\
\text { prisionais, passam por processos de assujeitamento, trazendo a ideia } \\
\text { de instituição punitiva. As ações médicas se limitam ao diagnóstico da } \\
\text { doença, onde as PPL chegam ao serviço de saúde em um estado clínico } \\
\text { grave, trazendo à tona a relação com a medicina curativa, do mesmo modo } \\
\text { encontra-se a subordinação do reconhecimento da doença ao saber médico, } \\
\text { o apenado não tem propriedade em identificar sinais e sintomas sugestivos } \\
\text { para TB. O atraso de medidas de prevenção e controle da doença se justificam } \\
\text { pelo contexto no qual as PPL estão inseridas, em uma instituição disciplinar, } \\
\text { redutora de autonomias, bem como em formações discursivas que definem } \\
\text { papéis (por construção social) e motivam visões acerca dos sujeitos no } \\
\text { interior da rede penitenciária. O artigo apresenta que no processo regulatório } \\
\text { do acesso das PPL sintomática para TB, as práticas assistenciais para o } \\
\text { diagnóstico da doença revelam linhas de poder envolvendo outras PPL e/ } \\
\text { ou agentes de segurança, que deliberam o acesso do apenado doente aos } \\
\text { serviços. Neste estudo, o presídio é identificado como um lugar de morte, de } \\
\text { sofrimentos, de preocupações. Ao hospital, unidade de referência da doença } \\
\text { na região do estudo, por sua vez, atribui-se a representação de lugar de vida, } \\
\text { saúde, cuidado. O estudo não deixa explícito como se dá o fluxo de acesso ao } \\
\text { serviço de saúde do apenado doente. }\end{array}$ \\
\hline
\end{tabular}

à reclusão, citados anteriormente ${ }^{(1)}$. Sendo a TB um grave problema de saúde pública, no que diz respeito ao controle de doenças infectocontagiosas, por seus altos níveis endêmicos registrados a nível mundial ${ }^{(6)}$ e por questões sociais, torna-se importante pensar em estratégias de prevenção e controle da TB no ambiente prisional a partir das PP recomendadas a nível nacional.

A abordagem dos artigos identificados na análise temática relaciona-se diretamente com as medidas de prevenção e controle da TB estabelecidas pelo MS no âmbito prisional. Segun- do a Organização Mundial da Saúde (OMS), devem ser elaboradas políticas para controle da infecção tuberculosa nas instituições prisionais 5. Procópio (2014) traz que para a implementação das PP são necessárias estruturas operacionais 14 , ou seja, lugares institucionais onde se ofertam serviços de saúde e ligações que os comuniquem em uma rede de atenção à saúde em todo território nacional 15, ofertadas universalmente ao conjunto da sociedade. A tradução dessa estrutura no Brasil é o Sistema Único de Saúde (SUS).

Entre as PP que tange às PPL desta- cam-se: o PNSSP, programa que garante o acesso à saúde das PPL, sua proposta é garantir ações integrais de saúde, além da assistência à prevenção e à promoção da saúde da população carcerária; a PNAISP, política que legitima a garantia ao direito à saúde para todas as PPL, visa a garantia do acesso dessa população ao SUS, respeitando os preceitos dos direitos humanos e de cidadania 4 , com a PNAISP toda unidade prisional habilitada pelo SUS passará a ser ponto de atenção da Rede de Atenção à Saúde; por último, a estrutura que organiza as ações de prevenção e controle da TB no 
país, o PNCT, tem por princípio diagnosticar e tratar precocemente os doentes, prevenir a TB primária e priorizar populações mais vulneráveis, como grupos populacionais com maior potencial social ou de risco biológico de adoecer

${ }^{14}$. Diante desse cenário torna-se importante compreender como têm sido desenvolvidas as PP e a implementação das mesmas nos presídios brasileiros.

Em relação à análise feita dos artigos selecionados para esta revisão integrativa, o artigo 1 apresenta que as ações de medidas de prevenção e controle da TB são parcialmente implementadas nas unidades prisionais brasileiras, destacando os aspectos favoráveis e desfavoráveis da implementação dessas PP voltadas às PPL. Ressalta-se que os estudos utilizaram diferentes instrumentos na implementação das ações. Foram analisadas 5 unidades de saúde prisionais, divididas entre Caso 1 e Caso 2. O estudo foi realizado em dois estados brasileiros não identificados e que apresentam altas taxas de incidência da doença. Foram consideradas somente unidades com detentos do sexo masculino, que compõem a grande maioria da população encarcerada e por ser o gênero com maior prevalência de $\mathrm{TB}{ }^{14}$. Evidencia-se que a implementação das PP não é realizada na íntegra nos dois casos apresentados, existem ainda diferenças entre a implementação das PP nos diferentes locais. Comparando os dois casos, observou-se que o Caso 1 apresenta ações favoráveis à implementação das medidas de prevenção e controle da doença, como a localização da unidade de saúde ser no mesmo pavilhão onde se encontram os detentos, a não necessidade de escolta para acessar o serviço de saúde, campanhas periódicas de busca ativa, exames de ingressos e de contato, oferta do teste anti-HIV, exames de cultura e TSA para casos diagnosticados e sintomáticos, notificação de casos, TDO, acompanhamento de casos por uma equipe multiprofissional e a garantia de medicamentos. Por outro lado, ainda no Caso 1, observa-se aspectos desfavoráveis, como a unidade de saúde
As PPL exemplificam, através de relatos que referem-se à presença

da TB entre os

apenados, percepção

da transmissão da

doença e ao modo de

interpretação para

a identificação da

mesma, que as açóes

médicas se limitam

ao diagnóstico da

doença, com os

apenados chegando

ao serviço de saúde

em um estado clínico

grave. Os mesmos

relatos trazem o

papel do poder

da administração

penitenciária

na regulação do

acesso do doente à

assistência de saúde. estar em condições precárias e radiografia extramuros para poucos casos.

O Caso 2 traz aspectos favoráveis, como uma unidade de saúde em boas condições, exames de baciloscopia de escarro, exames de radiografia, acompanhamento de casos por uma equipe específica, garantia de medicamentos e notificação de casos. No entanto, como aspecto desfavorável, e que diverge do Caso 1, traz a necessidade de escolta para acessar o serviço de saúde, carência de transporte até as unidades de saúde de referência, detecção passiva de casos e rastreamento radiológico interrompido, falta de recursos para oferta do anti-HIV, exame de cultura, TSA, da dispensação semanal de medicamentos, do tratamento auto administrado e equipe profissional não ativa no tratamento.

$\mathrm{O}$ artigo 2 apresenta na íntegra as condiçôes de saúde das PPL que vivem com TB e que são diagnosticadas com a doença tardiamente nos presídios brasileiros a partir da análise de múltiplos discursos de apenados. O estudo foi realizado no estado da Paraíba e, assim como no artigo anterior, foram incluídos somente detentos do sexo masculino. $\mathrm{O}$ texto atribui as condições de saúde dos apenados a circunstâncias favoráveis à disseminação de doenças infectocontagiosas como a TB, uma vez que os mesmos vivem em condições insalubres, contribuindo para as altas taxas de incidência e prevalência da doença se comparada com a população extramuros ${ }^{16}$.

As PPL exemplificam, através de relatos que referem-se à presença da $\mathrm{TB}$ entre os apenados, percepção da transmissão da doença e ao modo de interpretação para a identificação da mesma, que as ações médicas se limitam ao diagnóstico da doença, com os apenados chegando ao serviço de saúde em um estado clínico grave. Os mesmos relatos trazem o papel do poder da administração penitenciária na regulação do acesso do doente à assistência de saúde. Entretanto, o estudo não deixa explícito como se dá o fluxo de acesso ao serviço de saúde do apenado doente.

O PNSSP, a PNAISP e o PNCT reco- 
mendam ações de saúde semelhantes para o controle da TB no sistema prisional, como a busca de casos de TB, identificação de sintomático respiratório, exame de baciloscopia de escarro, exame de radiografia e TSA, tratamento imediato e supervisionado, oferta de teste anti-HIV, inscrição no Livro de Registro, notificação de casos, prevenção para as PPL sadias e o desenvolvimento de ações educacionais ${ }^{17,18}$. Diferentemente do PNSSP e da PNAISP, que se complementam e têm como objetivo a garantia do direito à saúde pelas PPL, o PNCT tem como objetivo reduzir a mortalidade, morbidade e transmissão da TB, implementando ações a nível nacional 14. As estratégias estabelecidas pelas PP citadas são identificadas no artigo 1 como fatores favoráveis na implementação da política. No entanto, no artigo 2 essas ações não são citadas de forma explícita, mas evidencia-se a partir das falas dos apenados que as mesmas não são implementadas na instituição de estudo.

O TDO nas unidades de análise do Caso 1 do artigo 1 era realizado nos dias úteis por um profissional de saúde. Nos fins de semana, a entrega do medicamento ficava sob a responsabilidade do agente de segurança. No Caso 2, a dispensação dos medicamentos era feita semanalmente por um profissional de saúde, sendo o tratamento auto administrado. $\mathrm{O}$ tratamento auto administrado também é mencionado pelos detentos do artigo 2, tornando-se evidente a não implementação do TDO, elemento chave no controle da TB, que consiste no profissional de saúde enxergar o paciente deglutir as medicações diariamente. Essa prática visa o fortalecimento da adesão do paciente ao tratamento, reduzindo casos de abandono e aumentando a probabilidade de cura 14. Esse elemento compõe as estratégias recomendadas pela OMS ${ }^{19}$, sendo um dos objetivos o tratamento padronizado com supervisão da equipe médica ou de enfermagem, a administração feita por agentes de segurança não é recomendada pelo PNCT ${ }^{14}$.

Evidenciam-se os diversos obstáculos
A pesquisa

identificou carência

de estudos relativos

a medidas de

prevenção e controle

da TB no contexto

carcerário brasileiro.

Pode-se inferir, que

a implementação

das PP relacionadas

às medidas de

prevenção e controle

da TB para as PPL

é realizada de forma

parcial. que dificultam a implementação de estratégias de controle da TB nas prisões brasileiras, destacando-se a subvalorização dos sinais e sintomas sugestivos para a doença, risco de estigmatização e segregação pelo reconhecimento da doença, falta de recursos humanos e financeiros, menos oportunidade de diagnóstico e tratamento, pouco acesso à informação sobre $\mathrm{TB}{ }^{5}$.

As PP garantem o acesso à saúde pelas PPL. Essas políticas devem contemplar todas as dimensões da saúde, especialmente a melhoria das condições de encarceramento e facultar o acesso a todos os apenados ${ }^{20}$. Torna-se necessário que essas ações sejam implantadas de maneira que atendam às PPL na porta de entrada do sistema, como também ao longo do cumprimento da pena ${ }^{14}$.

Ressalta-se que a garantia do acesso das PPL às ações e aos serviços de saúde constitui responsabilidade partilhada pelos Ministérios da Justiça (MJ) e da Saúde (MS), sua concretização implica efetiva parceria entre as Secretarias de Saúde e de Justiça/ Administração Penitenciária nos níveis estadual e municipal ${ }^{20}$.

\section{CONCLUSÃO}

A pesquisa identificou carência de estudos relativos a medidas de prevenção e controle da TB no contexto carcerário brasileiro. Pode-se inferir, que a implementação das PP relacionadas às medidas de prevenção e controle da TB para as PPL é realizada de forma parcial.

É contundente a existência de obstáculos para a implementação de forma efetiva das ações de saúde no sistema prisional, devido às más condições vida e de saúde das PPL, além das precárias condições de trabalho dos profissionais de saúde e de segurança. Ressalta-se a importância da perspectiva intersetorial entre o MS e MJ, respeitando as competências exclusivas de cada setor, a fim de garantir os direitos de saúde e manter os direitos humanos dos apenados, visando o aperfeiçoamento das estratégias voltadas à prevenção e ao controle da TB nesses estabelecimentos. - 


\section{REFERÊNCIAS}

1. Valença MS, Possuelo LG, Cezar-Vaz MR, Silva PEA. Tuberculose em presídios brasileiros: uma revisão integrativa da literatura. Ciência \& Saúde Coletiva. [Internet]. 2016 [citado 2018 set 4]; 21(7): 2147-2160. Disponível em: http://www.scielo.br/ $\mathrm{pdf} / \mathrm{csc} / \mathrm{v} 21 \mathrm{n} 7 / 1413-8123-\mathrm{csc}-21-07-2147 . \mathrm{pdf}$.

2. Sanchez A, Larouzé B. Controle da tuberculose nas prisões, da pesquisa à ação: a experiência do Rio de Janeiro, Brasil. Ciência \& Saúde Coletiva. [Internet]. 2016 [citado 2018 setembro 4]; 21(7): 2071-2079. Disponível em: http://www.scielo.br/pdf/ csc/v21n7/1413-8123-csc-21-07-2071.pdf.

3. Santos MN de A, Sá, AMM. Viver com tuberculose nas prisões: desafio de curar-se. Texto contexto Enferm. [Internet]. 2014 [citado 2018 set 4]; 23(4): 854-861. Disponivel em: http://www.scielo.br/pdf/tce/v23n4/pt_0104-0707tce-23-04-00854.pdf.

4. Lermen HS, Gil BL, Cúnico SD, Jesus LO de. Saúde no cárcere: análise das políticas sociais de saúde voltadas a população prisional brasileira. Rev. Saúde Coletiva. [Internet]. 2015 [citado 2018 set 8]; 25(3): 905-924. Disponivel em: http://www.scielo. br/pdf/physis/v25n3/0103-7331-physis-25-03-00905.pdf.

5. Ministério da Saúde (BR), Secretária de Vigilância em Saúde, Departamento de Vigilância Epidemiológica. Manual de recomendações para o controle da Tuberculose no Brasil [Internet]. Brasília; 2011 [citado 2018 set 4]. Disponível em: http:// bvsms.saude.gov.br/bvs/publicacoes/manual_recomendacoes_controle_tuberculose_brasil.pdf.

6. Ministério da Saúde (BR), Secretária de Vigilância em Saúde, Departamento de Vigilância Epidemiológica, Coordenação Geral de Doenças Endêmicas, Programa Nacional de Controle da Tuberculose. Plano Estratégico para o Controle da Tuberculose, Brasil 2007-2015 [Internet]. Brasília; 2006 [citado 2018 set 8]. Disponível em: https://www.paho. org/bra/index.php?option=com_docman\&view=download\&alias=927-plano-estrategico-para-o-controle-da-tuberculose-brasil-2007-2015-7\&category_slug=tuberculose-971\&ltemid=965.

7. Presidência da República (BR), Casa Civil, Subchefia para Assuntos Jurídicos. Lei n 7.210, de 11 de julho de 1984: institui a Lei de Execução Penal [Internet]. Diário Oficial da União da República Federativa do Brasil. 1984 jul 11 [citado 2018 set 4]. Disponivel em: http://www.planalto.gov.br/ccivil_03/LEIS/ L7210.htm.

8. Ministério da Saúde (BR), Gabinete do Ministro. Portaria interministerial $n^{\circ}$ 1.777, de 09 de setembro de 2003 [Internet]. Diário Oficial da União da República Federativa do Brasil. 2003 set 9 [citado 2018 set 8]. Disponivel em: http://bvsms.saude. gov.br/bvs/saudelegis/gm/2003/pri1777_09_09_2003.html.

9. Ministério da Saúde (BR), Gabinete do Ministro. Portaria interministerial $n^{\circ} 1$, de 2 de janeiro de 2014 [Internet]. Diário Oficial da União da República Federativa do Brasil. 2014 jan 2 [citado 2018 set 8]. Disponível em: http://bvsms.saude.gov.br/ bvs/saudelegis/gm/2014/pri0001_02_01_2014.html.
10. Mendes KDS, Silveira RCCP, Galvão CM. Revisão integrativa: método de pesquisa para a incorporação de evidências na saúde e na enfermagem. Texto e Contexto Enferm. [Internet]. 2008 [citado 2018 set 14]; 17(4): 758-764. Disponível em: http://www.scielo.br/pdf/tce/v17n4/18.pdf.

11. Júnior, LPC. A saúde atrás das grades: uma revisão bibliográfica buscando a importância do acesso a população carcerária ao sistema único de saúde. Ceilândia: UnB; 2015 [citado 2018 set 14]. Disponível em: http://bdm.unb.br/handle/10483/12642.

12. Oliveira LGS de, Natal S, Camacho LAB. Análise da implantação do Programa de Controle da Tuberculose em unidades prisionais no Brasil. Cad. Saúde Pública. [Internet]. 2015 [citado 2018 set 16]; 31(3): 543-554. Disponivel em: http://www. scielo.br/pdf/csp/v31n3/0102-311X-csp-31-03-00543.pdf.

13. Souza KMJ de, Villa TCS, Assolini FEP, Beraldo AA, França U de M, Protti ST et al. Atraso no Diagnóstico da Tuberculose no Sistema Prisional: a experiência do doente apenado. Contexto Enferm. [Internet]. 2012 [citado 2018 set 16]; 21(1): 17-25. Disponível em: http://www.scielo.br/pdf/tce/v21n1/a02v21n1. pdf.

14. Procópio MJ. Controle da tuberculose: uma proposta de integração ensino-serviço. 7 ed. rev. and enl. Rio de Janeiro: FIOCRUZ; 2014.

15. Ministério da Saúde (BR), Departamento de Atenção Básica. Elementos construtivos de Rede de Atenção à Saúde [Internet]. Brasília: Ministério da Saúde; 2018 [citado 2018 nov 17]. Disponivel em: http://dab.saude.gov.br/portaldab/smp_ras.php?conteudo=elementos_ras.

16. Macedo LR, Maciel ELN, Struchiner CJ. Tuberculose na população privada de liberdade do Brasil, 2007-2013. Epidemiol. Serv. Saúde. [Internet]. 2017 [citado 2018 set 19]; 26(4): 783794. Disponível: http://www.scielo.br/pdf/ress/v26n4/22379622-ress-26-04-00783.pdf.

17. Ministério da Saúde (BR), Gabinete do Ministro. Portaria $n^{\circ} 373$, de 27 de fevereiro de 2002 [Internet]. Diário Oficial da União da República Federativa do Brasil. 2002 fev 27 [citado 2018 nov 17]. Disponível em: http://bvsms.saude.gov.br/bvs/ saudelegis/gm/2002/prt0373_27_02_2002.html.

18. Ministério da Saúde (BR), Secretaria de Atenção à Saúde, Departamento de Ações Programáticas Estratégicas, Área Técnica de Saúde no Sistema Penitenciário. Plano Nacional de Saúde no Sistema Penitenciário [Internet]. Brasília: Ministério da Saúde; 2004 [citado 2018 nov 17]. Disponivel em: http:// bvsms.saude.gov.br/bvs/publicacoes/cartilha_pnssp.pdf.

19. World Health Organization. Global tuberculosis control 2018 [Internet]. Genebra: WHO; 2013 [citado 2018 set 17]. Disponivel em: http://www.who.int/tb/publications/global_report/en/.

20. Silva M. Saúde Penitenciária no Brasil: plano e política. Brasília: Verbena; 2015. 